\title{
ChemComm
}

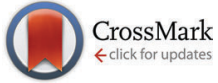

Cite this: Chem. Commun., 2015, 51, 16349

Received 25th July 2015,

Accepted 11th September 2015

DOI: $10.1039 / c 5 c c 06228 d$

www.rsc.org/chemcomm

\section{Real-time electrochemical monitoring of covalent bond formation in solution via nanoparticle- electrode collisions $\dagger$}

\author{
Da Li, $\ddagger^{a}$ Na Kong, $\ddagger^{a}$ Jingquan Liu, ${ }^{b}$ Hongbin Wang, ${ }^{c}$ Colin J. Barrow, ${ }^{a}$ \\ Shusheng Zhang ${ }^{c}$ and Wenrong Yang ${ }^{\star a}$
}

\begin{abstract}
We describe an alternative electrochemical technique to monitor covalent bond formation in real-time using nanoparticle-electrode collisions. The method is based on recognising the redox current when MP-11 functionalised chemical reduced graphene oxide (rGO) nanosheets collide with Lomant's reagent modified gold microelectrode. This facile and highly sensitive monitoring method can be useful for investigating the fundamental of single-molecule reactions.
\end{abstract}

We describe an alternative electrochemical technique to monitor single covalent bond formation in real-time via nanoparticleelectrode collision. In the past decades, work at the single-molecule level has attracted considerable attention because single-molecule studies can lead to important new insights about the effects of environment and configuration on the behavior of these molecules and such information is not available from ensemble studies. ${ }^{1,2}$ Researchers have measured covalent bond force using atom force microspectroscopy (AFM) ${ }^{3-5}$ However, there has been less emphasis on monitoring of covalent bond formation in real-time. Traditionally, covalent bonds can be monitored by high-resolution electron energy loss spectroscopy (HREELS), ${ }^{6}$ X-ray photoelectron spectroscopy (XPS), ${ }^{7}$ fluorescence, ${ }^{8}$ scanning tunnelling microscopy ${ }^{9}$ and femtosecond X-ray scattering. ${ }^{10}$ Collins and collaborators ${ }^{11-14}$ used point-functionalised carbon nanotubes device to continuously monitor a single carboxylate group interacting with $N$-ethyl- $N^{\prime}$ (3-dimethylaminopropyl)carbodiimidehydrochloride (EDC). This technique uses circuit conductance to monitor and control covalent attachment to an electrically connected single-walled carbon nanotubes. Discrete changes in the circuit conductance revealed chemical processes happening in real time. Although these progresses have been made, there are still challenges in high-resolution

\footnotetext{
${ }^{a}$ Center for Chemistry and Biotechnology, Deakin University, Victoria-3217,

Australia. E-mail: wenrong:yang@deakin.edu.au

${ }^{b}$ College of Chemical Science and Engineering, Qingdao University, Qingdao 266071, China

${ }^{c}$ Shandong Province Key Laboratory of Detection Technology for Tumor Makers, Linyi University, Linyi 276005, China

$\dagger$ Electronic supplementary information (ESI) available. See DOI: 10.1039/c5cc06228d

\$ These authors contributed equally to this work.
}

lithography and fabricating single-molecule electronic devices, and facile and sensitive alternatives are required.

The nanoparticle-electrode collision, a more efficient and low-cost electrochemical approach, is generally used for detecting various types of nanoparticles, such as metal particles, oxide particles, organic nanoparticles, a few molecules or even single molecule. ${ }^{15,16}$ Bard and coworkers ${ }^{17-19}$ reported the detection of metal nanoparticles (MNPs) through electrocatalytic amplification using a carbon ultramicroelectrode (UME) with Pt NPs in solution that was held at a constant potential, at which hydrogen evolution would occur on Pt but not on carbon. Previous studies demonstrated that there are two distinct types of reactivity: a cumulative cascade of current steps ("staircase") and a series of transiently decaying current jumps ("spikes"). A current staircase is expected for the permanent adsorption of nanoparticles. Current spikes are attributed to the hit-and-run nanoparticles for the limited time of residence. ${ }^{15,16}$ Previous studies also showed that a self-assembled monolayer (SAM) on electrodes can block the electron transfer tunneling to solution species. However, in the presence of MNP, more facile electron transfer (ET) can completely restore ET to solution species. The basis of this effect is that tunneling from the UME to a MNP is much more probable than tunneling to molecules in solutions. Suitable NPs might include, in addition to metals, semiconductor quantum dots, and carbon-based nanomaterials, such as graphene-oxide. ${ }^{20-23}$ Crooks and coworkers reported a real-time electrochemical monitoring of individual DNA hybridization events by monitoring electrocatalytic current when a complementary DNA strand labelled with a catalyst hybridizes to the working electrode modified with single-stranded DNA. ${ }^{24}$ In addition to detecting catalytic current associated with single nanoparticle collisions, Compton's group pioneered on the direct electrochemistry of single electro-active nanoparticles. They detected the Ag NPs by measuring the anodic current-time transient through a method coined anodic particle coulometry and they observed the random collision of single electro-active indigo nanoparticle onto a carbon microelectrode to generate a transient reductive Faradaic current that depended on the size of the nanoparticles, allowing the 
measurements of the size distribution of organic nanoparticles. ${ }^{25,26}$ Recently, we have developed a new electrochemical monitoring approach for ultrasensitive detection of protein molecules (microperoxidase MP-11), which are attached to the surface of graphene nanosheets. ${ }^{27}$ Microperoxidase (MP-11) is made by proteolytic digestion of cytochrome $\mathrm{c}$ and consists of 11 amino residues which has been widely used for electrode modification as a redox enzyme. ${ }^{28,29}$ The non-covalently functionalized rGO nanosheets exhibited enhanced electroactive surface area, where MP-11 could produce amplified redox current when rGO nanosheets collide with the electrode, leading to the amplification of redox current. When the rGO nanosheet collides with the gold electrode it will become a nanoelectrode for a millisecond period. The approach provides a novel platform to fabricate a biosensor using nano rGO. It provides a new tool for investigating individual chemical reactions at the singlemolecule level.

Herein, we demonstrated real-time monitoring of covalent bond formation in solution by nanoparticle-electrode collisions. To monitor covalent bond formation, we design two different nanoparticle-electrode collisions, Hit-and-Run and Hit-and-Stand (Fig. 1). A single layer Lomant's reagent (an ester with NHS) was coated on the surface of the gold electrode. Once MP-11/rGO nanosheets reach the surface of Lomant's reagent coated gold electrode, an amido covalent bond was formed via the $\mathrm{NH}_{2}$ group from the MP-11 and carboxyl from the Lomant's reagent (Fig. 2). Then MP-11/rGO nanosheets stick onto the gold electrode surface, rather than being repelled away. The collision process is a Hit-andStand one. As a control experiment, the gold electrode was coated with a SAM of alkane thiols (3-mercaptopropanoic acid, MPA) terminated with carboxyl group. When MP-11/rGO nanosheets were diffused to the surface of gold electrode coated with SAM, it was repelled away by the electrostatic repulsive force because MP-11/ rGO nanosheets are negatively charged and the SAMs are also negatively charged due to the carboxyl groups. This collision is a Hit-and-Run process. The current changes during Hit-andStand process show the collision behavior of MP-11/rGO when it reaches and sticks on the electrode permanently through covalent bonds. This collision behavior of MP-11/rGO is different from that on MPA modified electrode where MP-11/rGO is

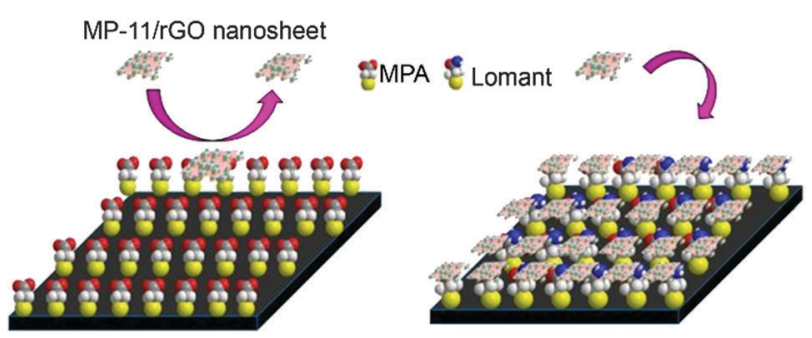

(a) Electrostatic interaction (Hit-n-Run) (b) Covalent bond (Hit-n-Stand)

Fig. 1 Schematic of monitoring covalent bond formation. (a) Non covalent bond (electrostatic interaction), (b) covalent bond. The amplified redox current from MP-11 can be monitored. An amido covalent bond forms when MP-11 functionalised $\mathrm{rGO}$ nanosheets reach Lomant's reagent (SAMs) modified gold electrode and stick on the SAMs. The amplified redox current from MP-11 was detected.
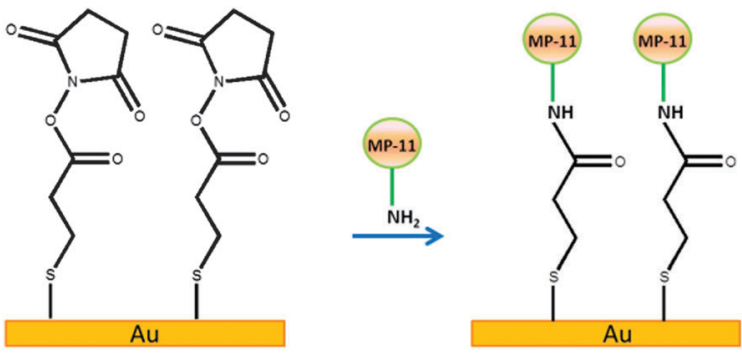

Fig. 2 Schematic of covalent bond formation. The amido covalent bonds form when MP-11 functionalised $\mathrm{rGO}$ nanosheets reach Lomant's reagent modified gold electrode and stick on the SAM.

repelled with electrostatic interaction. Therefore MP-11/rGO collision with Lomant's reagent modified electrode could be used to indirectly monitor covalent bond formation.

The rGO nanosheets were synthesized by modified Hummer's method. ${ }^{22,30,31}$ The preparation and characterization processes of Lomant's reagent and MPA modified gold electrodes were provided in detail in ESI $\dagger$ (Fig. S2 and S3). The modified electrodes were denoted as Lomant/Au and MPA/Au, respectively.

To determine the redox potential of MP-11/rGO nanosheets, cyclic voltammetry was performed (Fig. S4, ESI $\dagger$ ). The reduction potential of MP-11 is about $-0.4 \mathrm{~V}$. The threshold potential used for MP-11 collision experiment is $-0.4 \mathrm{~V}$. To monitor the formation of covalent bonds, chronoamperograms of Lomant/Au electrode and MPA/Au electrode $(12.5 \mu \mathrm{m})$ were recorded separately after injection of MP-11/rGO nanosheets in $10 \mathrm{mM}$ PBS buffer solution $(\mathrm{pH}=7)$. When the MP-11/rGO nanosheets collided with the electrode surface, current profiles were observed at $-0.4 \mathrm{~V}$ or above for both Lomant's and MPA modified electrodes. The redox current of MP-11 that amplified when MP-11 is assembled on rGO nanosheets. ${ }^{27}$ The redox current of the Lomant/Au electrode showed a stair case response, however, the MPA/Au electrode showed a spike response (Fig. 3a). When MP-11/rGO nanosheets land in the Lomant's electrode surface, the amplified redox current reflects attachments of MP-11/rGO nanosheets via carbodiimide activated amidation reaction between the Lomant's reagent terminal groups and the amino functionalities of MP- $11 / \mathrm{rGO}$ nanosheets. ${ }^{28} \mathrm{MP}-11 / \mathrm{rGO}$ nanosheets are anchored to the electrode surface and the redox current is simultaneously amplified and shows a staircase response.

It is clear that the stepwise current-time response indicated that MP-11 sticks to the electrode upon contact, once MP-11/rGO nanosheets land in the Lomant's electrode surface. Based on control experiments, the permanent attachment of MP-11/rGO nanosheets strongly suggested covalent bond formation via carbodiimide activated amidation reaction between the Lomant's reagent terminal groups and the amino functionalities of MP-11/rGO nanosheets. The current increase is the synergetic results of MP-11/rGO nanosheets. The number of staircase steps indicated the number of attached MP$11 / \mathrm{rGO}$ nanosheets to the Lomant/Au electrode surface. The attached MP-11/rGO nanosheets are hold by covalent bonds between MP-11/ rGO nanosheets the Lomant/Au electrode surface. The total binding strength will depend on the number of covalent bonds. For the MPA/Au electrode, only spike response was observed. The electrode 
(a)

(b)

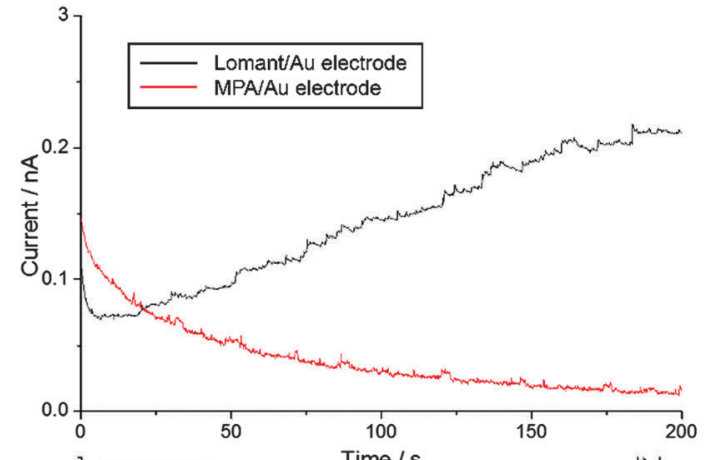

(c)

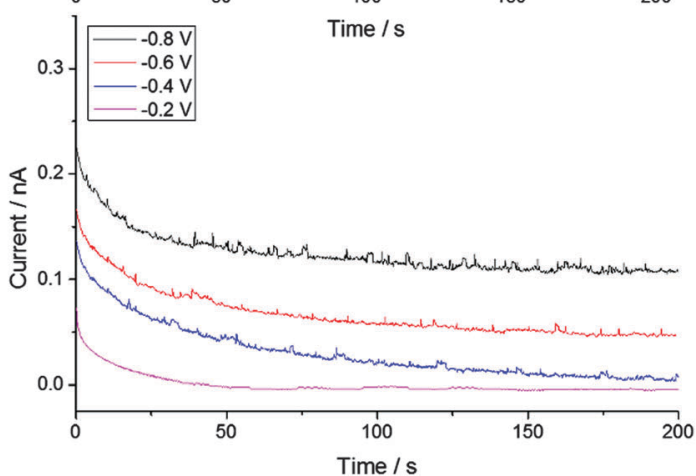

Fig. 3 Current transient recorded at $-0.4 \mathrm{~V}$ after injection of MP-11/rGO nanosheets at Lomant/Au and MPA/Au electrode (diameter, $12.5 \mu \mathrm{m}$ ), respectively (a). Current transient recorded after injection of MP-11/rGO nanosheet at Lomant/Au (b) and MPA/Au (c) at different potential.

surface is negatively charged due to the terminated carboxyl group, while the MP-11/rGO nanosheet is also negatively charged, and so there is an electrostatic repulsion between them. Furthermore, please note when the MP-11/rGO nanosheets collide with MPA/ $\mathrm{Au}$ electrode, this collision process was the Hit-and-Run case. In our previous work, ${ }^{27}$ once MP-11/rGO nanosheets collide with bare gold electrode, current spikes were observed at potentials above $-0.4 \mathrm{~V}$, which also indicated Hit-and-Run nanoparticles would contribute a current spike due to the limited time of residence.

Fig. $3 \mathrm{~b}$ and $\mathrm{c}$ show that current increases with the various potentials at both Lomant/Au and MPA/Au electrodes. The current spikes was dependent on the reduction potential. No spikes were found when the potential was below $-0.4 \mathrm{~V}$. As for control experiments, no current spikes were observed for the Lomant's reagent electrode and MPA electrode in $10 \mathrm{mM}$ PBS $(\mathrm{pH}=7.0)$ at a potential range of -0.2 to $-0.8 \mathrm{~V}$ after injection of MP-11 (Fig. S5, ESI $\dagger$ ). The average charge passed each spike can be calculated by dividing the total charge with the total spike number at a given potential. The average charge passed each spike produced by MP-11/rGO at Lomant/Au and MPA/Au electrode at different potential was analyzed in Fig. 4a. The average charge passed each spike increases with the increase of potential. More negative potential may increase the electron transfer rate and hence more charge is transferred for each collision event. The MP-11/rGO nanosheet collided with and was removed quickly from the gold electrode. The MP-11/rGO nanosheets stayed at the gold surface for milliseconds after the collision. ${ }^{32}$ The MP-11 is positive charged because of the $\mathrm{Fe}^{3+}$ ion. The rGO nanosheet is negative charged owing to the negative charged functional groups such as $-\mathrm{COO}^{-}$. The MP$11 /$ rGO is weakly negatively charged. The zeta potential of MP-11, rGO and MP-11/rGO is $15 \pm 3 \mathrm{mV},-30 \pm 5 \mathrm{mV}$ and $-11 \pm 2 \mathrm{mV}$ at $\mathrm{pH}$ of 7.0. So the electrophoresis is almost negligible for the potential dependent collision. Fig. 4b shows the variation of spike frequency with concentration of MP-11/ rGO at Lomant/Au and MPA/Au electrode, respectively. The collision frequency was also recorded in Fig. 4c at different potentials after injection of MP-11/rGO nanosheet, at Lomant/Au and MPA/Au electrode, respectively. The spike frequency increases with increasing MP-11/rGO concentration for Lomant/Au and MPA/ Au electrodes.

At a higher concentration, more redox reaction of MP-11 occurs, which induces the higher spike frequency. Even at the same concentration, the spike frequency of Lomant/Au electrode is higher than that of MPA/Au electrode, which could be attributed to the electrostatic repulsion that caused less collision events between the both negatively charged MPA/Au electrode surface and MP-11/rGO nanosheets. Fig. 4d shows that the staircase/spike height increased with the increased applied potentials for both Lomant/Au and MPA/Au electrodes. The current transient at Lomant/Au electrode ranges in 9.6-22 pA, while at MPA/Au electrode it is in the range of 6.2-14 pA that obviously lower than that of Lomant/Au electrode, which might reflect the Lomant/Au electrodes can facilitate faster electron transfer than MPA/Au electrodes.

We attempt to estimate the number of covalent bonds formed during the collision based on the staircase current. The charge passed each spike is related to the number of electrons passed, which could be used to calculate the number of MP-11 molecules on both sides of rGO sheet. The average charge of each spike of MP-11/rGO nanosheet at $-0.4 \mathrm{~V}$ at Lomant/Au electrode is $0.31 \mathrm{pC}$. In our experiment, the total charge transfer during each collision event are induced by the redox reaction in MP-11 active center $\left(\mathrm{Fe}^{3+} / \mathrm{Fe}^{2+}\right)$. Lemay et al. ${ }^{33}$ monitored 8-46 enzyme molecules based on a maximum turnover rate of 1500 to $9000 \mathrm{~s}^{-1}$ for their case. Based on the average charge of each spike of MP-11/rGO, we estimated the number of MP-11 molecules on a single rGO nanosheet was $217 \pm 36 .{ }^{27}$ We also realized it is a challenge to determine the number of covalent bonds of amino group on MP-11/rGO sheets during the collision process. There are 11 amino residues in a MP-11 molecule. If we assume that the reaction rate of amino group is $50 \%$, then an average value of $596 \pm 99$ covalent bonds on a single MP-11/rGO nanosheet was roughly estimated. 
(a)

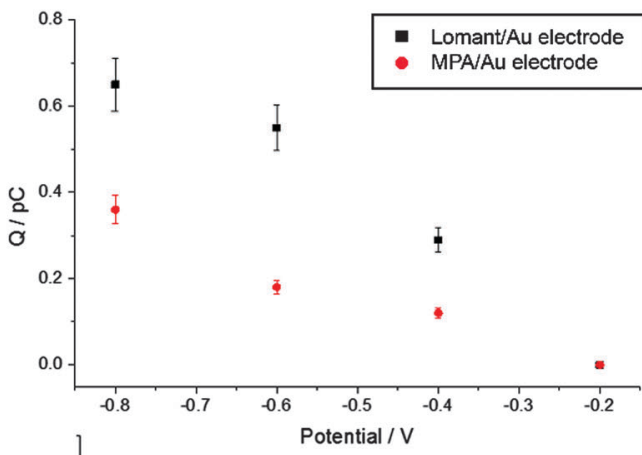

(b)

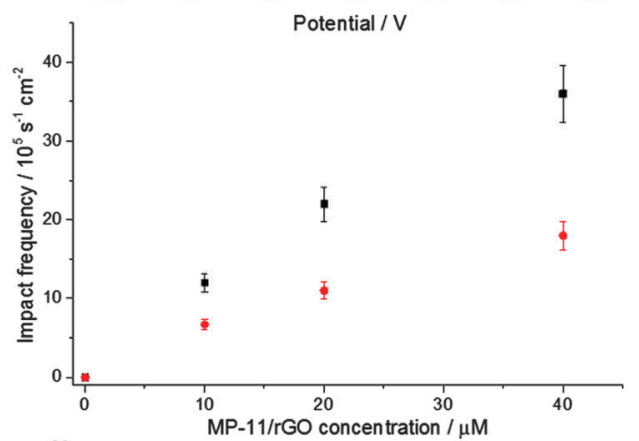

(c)

(d)
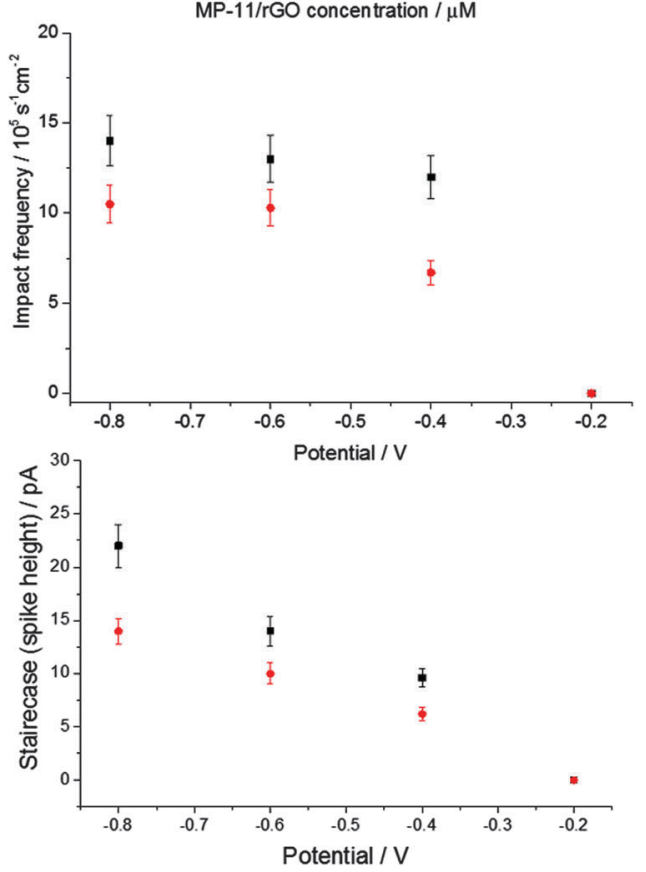

Fig. 4 (a) The average charge passed each spike produced by MP-11/rGO nanosheets at different potentials. (b) The collision frequency recorded with different MP-11 concentrations. (c) The collision frequency recorded at different potentials. (d) The staircase/spike height changes with potentials.

Lomant/Au electrode,

MPA/Au electrode, $12.5 \mu \mathrm{m}$ ).

In conclusion, we have demonstrated a new electrochemical technique to monitor covalent bond formation in real-time via nanoparticle-electrode collisions using the amplified redox current MP-11 functionalized rGO nanosheet, which is anchored on the electrode by covalently attachments of MP-11/rGO nanosheets via carbodiimide activated amidation reaction between the Lomant's reagent terminal groups and the amino functionalities of MP-11/rGO nanosheets. This facile and highly sensitive monitoring method could be useful for investigating the fundamental of single-molecule reactions.

\section{Notes and references}

1 Y. Fang, W. Wang, X. Wo, Y. Luo, S. Yin, Y. Wang, X. Shan and N. Tao, J. Am. Chem. Soc., 2014, 136, 12584.

2 A. J. Bard, ACS Nano, 2008, 2, 2437.

3 X. Hao, N. Zhu, T. Gschneidtner, E. Ö. Jonsson, J. Zhang, K. MothPoulsen, H. Wang, K. S. Thygesen, K. W. Jacobsen and J. Ulstrup, Nat. Commun., 2013, 4, 2121.

4 S. S. Wong, E. Joselevich, A. T. Woolley, C. L. Cheung and C. M. Lieber, Nature, 1998, 394, 52.

5 M. Grandbois, M. Beyer, M. Rief, H. Clausen-Schaumann and H. E. Gaub, Science, 1999, 283, 1727.

6 T. Yamada, T. Inoue, K. Yamada, N. Takano, T. Osaka, H. Harada, K. Nishiyama and I. Taniguchi, J. Am. Chem. Soc., 2003, 125, 8039.

7 K. Bell, P. Brooksby, M. Polson and A. Downard, Chem. Commun., 2014, 50, 13687.

8 S. Klüter, J. R. Simard, H. B. Rode, C. Grütter, V. Pawar, H. C. Raaijmakers, T. A. Barf, M. Rabiller, W. A. van Otterlo and D. Rauh, ChemBioChem, 2010, 11, 2557.

9 A. Ciesielski, M. El Garah, S. Haar, P. Kovaříček, J.-M. Lehn and P. Samorì, Nat. Chem., 2014, 6, 1017.

10 K. H. Kim, J. G. Kim, S. Nozawa, T. Sato, K. Y. Oang, T. W. Kim, H. Ki, J. Jo, S. Park, C. Song, T. Sato, K. Ogawa, T. Togashi, K. Tono, M. Yabashi, T. Ishikawa, J. Kim, R. Ryoo, J. Kim, H. Ihee and S.-I. Adachi, Nature, 2015, 518, 385.

11 B. R. Goldsmith, J. G. Coroneus, V. R. Khalap, A. A. Kane, G. A. Weiss and P. G. Collins, Science, 2007, 315, 77.

12 B. R. Goldsmith, J. G. Coroneus, A. A. Kane, G. A. Weiss and P. G. Collins, Nano Lett., 2008, 8, 189.

13 Y. Choi, T. J. Olsen, P. C. Sims, I. S. Moody, B. L. Corso, M. N. Dang, G. A. Weiss and P. G. Collins, Nano Lett., 2013, 13, 625.

14 Y. Choi, I. S. Moody, P. C. Sims, S. R. Hunt, B. L. Corso, I. Perez, G. A. Weiss and P. G. Collins, Science, 2012, 335, 319.

15 S. E. Kleijn, S. Lai, M. Koper and P. R. Unwin, Angew. Chem., Int. Ed., $2014,53,3558$.

16 W. Wang and N. Tao, Anal. Chem., 2013, 86, 2.

17 X. Xiao, S. Pan, J. S. Jang, F.-R. F. Fan and A. J. Bard, J. Phys. Chem. C, 2009, 113, 14978.

18 J. E. Dick, C. Renault, B. K. Kim and A. J. Bard, Angew. Chem., Int. Ed., 2014, 126, 12053-12056.

19 B.-K. Kim, A. Boika, J. Kim, J. E. Dick and A. J. Bard, J. Am. Chem. Soc., 2014, 136, 4849.

20 J.-N. Chazalviel and P. Allongue, J. Am. Chem. Soc., 2010, 133, 762.

21 A. Chou, P. K. Eggers, M. N. Paddon-Row and J. J. Gooding, J. Phys. Chem. C, 2009, 113, 3203.

22 B. Zhang, L. Fan, H. Zhong, Y. Liu and S. Chen, J. Am. Chem. Soc., 2013, 135, 10073.

23 J. Kim, B.-K. Kim, S. K. Cho and A. J. Bard, J. Am. Chem. Soc., 2014, 136, 8173.

24 T. M. Alligrant, E. G. Nettleton and R. M. Crooks, Lab Chip, 2013, 13, 349.

25 Y. G. Zhou, N. V. Rees and R. G. Compton, Angew. Chem., Int. Ed., $2011,50,4219$.

26 W. Cheng, X. F. Zhou and R. G. Compton, Angew. Chem., 2013, 125,13218

27 D. Li, J. Liu, C. J. Barrow and W. Yang, Chem. Commun., 2014, 50, 8197.

28 J. J. Gooding, R. Wibowo, J. Liu, W. Yang, D. Losic, S. Orbons, F. J. Mearns, J. G. Shapter and D. B. Hibbert, J. Am. Chem. Soc., 2003, $125,9006$.

29 T. Ruzgas, A. Gaigalas and L. Gorton, J. Electroanal. Chem., 1999, 469, 123.

30 S. William, J. Hummers and R. Offeman, J. Am. Chem. Soc., 1958, 80, 1339.

31 J. Zhang, H. Yang, G. Shen, P. Cheng, J. Zhang and S. Guo, Chem. Commun., 2010, 46, 1112.

32 J. M. Kahk, N. V. Rees, J. Pillay, R. Tshikhudo, S. Vilakazi and R. G. Compton, Nano Today, 2012, 7, 174.

33 F. J. Hoeben, F. S. Meijer, C. Dekker, S. P. Albracht, H. A. Heering and S. G. Lemay, ACS Nano, 2008, 2, 2497. 